Supporting Information

\title{
Interaction of differently sized, shaped, and functionalized silver and gold nanoparticles with glycosylated vs. non-glycosylated transferrin
}

Rinea Barbira, Rafael Ramírez Jiménez ${ }^{\mathrm{b}}$, Rafael Martín-Rapún ${ }^{\mathrm{b}}$, Vida Strasserc ${ }^{\mathrm{c}}$, Darija Domazet Jurašin ${ }^{\mathrm{c}}$, Sanja Dabelićd ${ }^{\mathrm{d}}$ Jesus Martinez de la Fuente ${ }^{\mathrm{b}}$, Ivana Vinković Vrček ${ }^{\mathrm{a}, 1}$

a Institute for Medical Research and Occupational Health, Ksaverska cesta 2, 10000 Zagreb, Croatia

b Instituto de Nanociencia y Materiales de Aragón (INMA), Universidad de Zaragoza - CSIC and CIBER-BBN, Zaragoza, Spain

c Division of Physical Chemistry, Ruđer Bošković Institute, Bijenička cesta 54, 10000 Zagreb, Croatia

${ }^{\mathrm{d}}$ University of Zagreb, Faculty of Pharmacy and Biochemistry, Ante Kovačića 1, 10000 Zagreb, Croatia

${ }^{1}$ Corresponding author: ivinkovic@imi.hr 


\section{CONTENT:}

\section{Information on the commercial proteins used in the study.}

Table S1. The values of Stern-Volmer quenching constants $\left(K_{S V}\right)$ and quenching constants $\left(k_{q}\right)$ obtained from the interaction between different silver and gold nanomaterials (NMs) with bovine serum albumin (BSA), transferrin (TRF) and non-glycosylated transferrin (ngTRF).

Table S2. The logarithmic values of binding constants $\left(\log K_{\mathrm{b}}\right)$ and Hills coefficients $(n)$ obtained for the interaction of different silver and gold nanomaterials (NMs) with bovine serum albumin (BSA), human transferrin (TRF) and non-glycosylated recombinant transferrin (ngTRF).

Table S3. The Pearson correlation coefficients obtained from the statistical analysis of experimental data.

Figure S1. Fluorescence emission spectra of (a) bovine serum albumin (BSA), (b) transferrin (TRF) and (c) non-glycosylated transferrin (ngTRF), obtained at the protein concentration of 0.2 $\mu \mathrm{M}$ in the absence and presence of GSHAuNPs in concentration range of $0.01-0.18 \mathrm{nM}$. SternVolmer plots of fluorescence quenching at $0.2 \mu \mathrm{M}$ (d) BSA (e) TRF and (f) ngTRF by GSHAuNP and corresponding logarithmic plots of the fluorescence quenching of (g) BSA (h) TRF and (i) ngTRF.

Figure S2. Native conformation of bovine serum albumin (BSA), transferrin (TRF) and nonglycosylated transferrin (ngTRF) recorded using circular dichroism (CD) method.

Figure S3. Circular dichroism spectra of bovine serum albumin (BSA), transferrin (TRF) and nonglycosylated transferrin (ngTRF) in the absence and presence of mPEGAuNPrs $(a, b, c)$ and $\operatorname{lCITAuNP}(\mathrm{d}, \mathrm{e}, \mathrm{f})$ at the nanomaterials concentrations of $10 \mu \mathrm{M}, 50 \mu \mathrm{M}$ and $100 \mu \mathrm{M}$.

Figure S4. Changes in structural features of (a) bovine serum albumin (BSA), (b) transferrin (TRF) and (c) non-glycosylated transferrin (ngTRF) due to the interaction with different silver and gold nanomaterials at protein concentration of $100 \mu \mathrm{M}$ compared to the native protein.

Figure S5. Correlation between delta beta sheets of non-glycosylated TRF (\%) and specific surface area $\left(\mathrm{m}^{2} \mathrm{~g}^{-1}\right)$ of spherical NPs. 


\section{Information on the commercial proteins used in the study.}

As model proteins, commercially available bovine serum albumin (BSA), non-glycosylated human recombinant transferrin (ngTRF) and glycosylated human transferrin (TRF) were used. The ngTRF and TRF were purchased from ProSpec-Tany TechnoGene Ltd. International, East Brunswick NJ 08816, USA under catalogue numbers PRO-747 and PRO-315, respectively, while BSA was purchased from Sigma-Aldrich Chemie Gmbh (Munich, Germany) under catalogue number A7906.

Detailed description of ngTRF: Recombinant Human Transferrin produced in Plant is a nonglycosylated, polypeptide chain containing 679 amino acids and having a molecular mass of 76 $\mathrm{kDa}$. The Recombinant Human Transferrin is purified by proprietary chromatographic techniques. Synonyms: Serotransferrin, Transferrin, Siderophilin, Beta-1-metal-binding globulin, TF, PRO1557, PRO2086, DKFZp781D0156, HTF.

Source: Oryza sativa (rice).

Physical Appearance: Sterile Filtered lyophilized (freeze-dried) powder.

Formulation: The protein $(1 \mathrm{mg} / \mathrm{ml})$ was lyophilized with no additives.

Solubility: Stock solutions can be prepared by dissolving gently into PBS for several minutes. Recommended stock concentrations are $5 \mathrm{mg} / \mathrm{ml}$ to $20 \mathrm{mg} / \mathrm{ml}$ in PBS, though others can be used as well. Please try to avoid the formation of bubbles when dissolving the protein. Sterile filter through $0.2 \mu \mathrm{m}$ filter.

Stability: Lyophilized Transferrin although stable at room temperature for 3 weeks, should be stored desiccated below $-18^{\circ} \mathrm{C}$. Upon reconstitution Transferrin Human Recombinant should be stored at $4{ }^{\circ} \mathrm{C}$ between 2-7 days and for future use below $-18^{\circ} \mathrm{C}$. For long term storage it is recommended to add a carrier protein $(0.1 \%$ HSA or BSA). Purity as determined by SDS-PAGE is $97 \%$.

Iron Content: The average amount of Ferric Iron in our Transferrin is $0.4-0.5$ ug per mg of protein (protein concentration is measured by absorption at $280 \mathrm{~nm}$ ).

Biological Activity: One mg of Recombinant Human Transferrin will bind to approximately 2 micrograms of Fe. 
Detailed description of $n g T R F$ : Human Holo Transferrin is a glycoprotein of approximately 77 $\mathrm{kDa}$.

Synonyms: Serotransferrin, Transferrin, Siderophilin, Beta-1-metal-binding globulin, TF, PRO1557, PRO2086, DKFZp781D0156, Holo Transferrin, HTF.

Source: Human serum.

Physical Appearance: Sterile Filtered Pink lyophilized (freeze-dried) powder.

Formulation: The protein $(10 \mathrm{mg} / \mathrm{ml})$ was lyophilized from $20 \mathrm{mM}$ NH4HC03 solution. May contain traces of buffer salts.

Solubility: It is recommended to reconstitute the lyophilized Holo Transferrin in sterile $18 \mathrm{M} \Omega-\mathrm{cm}$ $\mathrm{H} 2 \mathrm{O}$ not less than $100 \mu \mathrm{g} / \mathrm{ml}$, which can then be further diluted to other aqueous solutions.

Stability: Store the lyophilized Holo Transferrin between $2-8^{\circ} \mathrm{C}$, do not freeze. Upon reconstitution Apo Transferrin should be stored at $4^{\circ} \mathrm{C}$ between $2-7$ days and for future use below $-18^{\circ} \mathrm{C}$.

Iron Content: The Iron content was estimated by ICP and was found to be $1232 \mathrm{ppm}$.

Purity: Greater than $98.0 \%$ as determined by coomassie blue stained SDS-PAGE and Cellulose Acetate electrophoresis.

Human Virus Test: FDA approved Plasma from each donor has been tested and found negative for antibodies to HIV-1 \& 2, HCV, HBsAG, HBc, HBV, HAV, HIV and Syphilis.

Detailed description of BSA: heat shock fraction, $\mathrm{pH} 7, \geq 98 \%$; CAS Number 9048-46-8; EC Number 232-936-2; MDL number MFCD00130384.

Synonym: Albumin bovine serum, BSA, Bovine albumin

Biological source: bovine

Assay: $\geq 98 \%$

Form: lyophilized powder

Mol wt. $\sim 66 \mathrm{kDa}$

Purified by: heat shock fractionation

Packaging: poly bottle of

Origin: USA origin

Loss $\leq 5 \%$; pH 7

Solubility: water: soluble $(40 \mathrm{mg} / \mathrm{ml})$

Storage temp. $2-8^{\circ} \mathrm{C}$

Gene Information: bovine ... ALB(280717) 
Table S1. The values of Stern-Volmer quenching constants $\left(K_{\mathrm{SV}}\right)$ and quenching constants $\left(k_{\mathrm{q}}\right)$ obtained from the interaction between different silver and gold nanomaterials (NMs) with bovine serum albumin (BSA), transferrin (TRF) and non-glycosylated transferrin (ngTRF). The results are presented as the mean of $K_{\mathrm{SV}}$ values calculated from nanoparticles concentration of three independent measurements.

\begin{tabular}{lllllll}
\hline \multirow{2}{*}{ NMs type } & \multicolumn{2}{c}{ BSA } & \multicolumn{2}{c}{ TRF } & \multicolumn{2}{c}{ ngTRF } \\
\hline GSHAgNP & $\boldsymbol{K}_{\mathbf{S v}} / \mathbf{M}^{-1}$ & $\boldsymbol{k}_{\mathbf{q}} / \mathbf{M}^{-1} \mathbf{s}^{-1}$ & $\boldsymbol{K}_{\mathbf{S v}} / \mathbf{M}^{-1}$ & $\boldsymbol{k}_{\mathbf{q}} / \mathbf{M}^{-1} \mathbf{s}^{-1}$ & $\boldsymbol{K}_{\mathbf{S V}} / \mathbf{M}^{-1}$ & $\boldsymbol{k}_{\mathbf{q}} / \mathbf{M}^{-1} \mathbf{s}^{-1}$ \\
\hline GSHAuNP & $3.50 \times 10^{7}$ & $7.00 \times 10^{15}$ & $3.00 \times 10^{7}$ & $6.00 \times 10^{15}$ & $2.00 \times 10^{7}$ & $4.00 \times 10^{15}$ \\
\hline sPVPAgNP & $8.67 \times 10^{7}$ & $1.73 \times 10^{16}$ & $3.67 \times 10^{7}$ & $7.33 \times 10^{15}$ & $4.33 \times 10^{7}$ & $8.67 \times 10^{15}$ \\
\hline lPVPAgNP & $2.67 \times 10^{8}$ & $5.33 \times 10^{16}$ & $1.67 \times 10^{8}$ & $3.33 \times 10^{16}$ & $9.33 \times 10^{7}$ & $1.87 \times 10^{16}$ \\
\hline CITAgNP & $1.67 \times 10^{8}$ & $3.33 \times 10^{16}$ & $2.33 \times 10^{8}$ & $4.67 \times 10^{16}$ & $5.00 \times 10^{8}$ & $1.00 \times 10^{17}$ \\
\hline sCITAuNP & $7.33 \times 10^{8}$ & $1.47 \times 10^{17}$ & $6.33 \times 10^{8}$ & $1.27 \times 10^{17}$ & $7.67 \times 10^{8}$ & $1.53 \times 10^{17}$ \\
\hline mCITtAuNP & $4.40 \times 10^{11}$ & $8.80 \times 10^{19}$ & $8.33 \times 10^{9}$ & $1.67 \times 10^{18}$ & $1.33 \times 10^{10}$ & $2.67 \times 10^{18}$ \\
\hline 1CITAuNP & $2.33 \times 10^{9}$ & $4.67 \times 10^{17}$ & $1.67 \times 10^{9}$ & $3.33 \times 10^{17}$ & $1.27 \times 10^{9}$ & $2.53 \times 10^{17}$ \\
\hline PEGAgNP & $4.33 \times 10^{8}$ & $8.67 \times 10^{16}$ & $3.33 \times 10^{8}$ & $6.67 \times 10^{16}$ & $2.67 \times 10^{8}$ & $5.33 \times 10^{16}$ \\
\hline PEGAuNP & $1.00 \times 10^{10}$ & $2.00 \times 10^{18}$ & $9.67 \times 10^{9}$ & $1.93 \times 10^{18}$ & $1.00 \times 10^{10}$ & $2.00 \times 10^{18}$ \\
\hline PEGAuNRs & $4.67 \times 10^{9}$ & $9.33 \times 10^{17}$ & $2.33 \times 10^{9}$ & $4.67 \times 10^{17}$ & $4.33 \times 10^{9}$ & $8.67 \times 10^{17}$ \\
\hline sPEGAuNPrs & $2.00 \times 10^{10}$ & $4.00 \times 10^{18}$ & $2.00 \times 10^{10}$ & $4.00 \times 10^{18}$ & $2.00 \times 10^{10}$ & $4.00 \times 10^{18}$ \\
\hline mPEGAuNPrs & $2.67 \times 10^{10}$ & $5.33 \times 10^{18}$ & $1.43 \times 10^{10}$ & $2.87 \times 10^{18}$ & $1.40 \times 10^{10}$ & $2.80 \times 10^{18}$ \\
\hline 1PEGAuNPrs & $1.03 \times 10^{11}$ & $2.07 \times 10^{19}$ & $2.23 \times 10^{10}$ & $4.47 \times 10^{18}$ & $1.00 \times 10^{11}$ & $2.00 \times 10^{19}$ \\
\hline
\end{tabular}


Table S2. The logarithmic values of binding constants $\left(\log K_{\mathrm{b}}\right)$ and Hills coefficients $(n)$ obtained for the interaction of different silver and gold nanomaterials (NMs) with bovine serum albumin (BSA), human transferrin (TRF) and non-glycosylated recombinant transferrin (ngTRF). Results are presented as a mean values obtained from 3 independent measurements with corresponding standard deviations (SD). Abbreviations of all NMs are explained in Table 1.

\section{$\begin{array}{lll}\text { BSA } & \text { TRF } & \text { ngTRF }\end{array}$}

\begin{tabular}{lcccccc} 
NMs type & $\log \boldsymbol{K}_{\mathbf{b}} \pm \mathbf{S D}$ & $\boldsymbol{n} \pm \mathbf{S D}$ & $\log \boldsymbol{K}_{\mathbf{b}} \pm \mathbf{S D}$ & $\boldsymbol{n} \pm \mathbf{S D}$ & $\log \boldsymbol{K}_{\mathbf{b}} \pm \mathbf{S D}$ & $\boldsymbol{n} \pm \mathbf{S D}$ \\
\hline GSHAgNPs & $5.32 \pm 0.05$ & $0.70 \pm 0.06$ & $8.49 \pm 0.40$ & $1.15 \pm 0.23$ & $11.44 \pm 0.12$ & $1.54 \pm 0.09$ \\
\hline GSHAuNPs & $12.10 \pm 0.31$ & $1.19 \pm 0.01$ & $13.55 \pm 0.81$ & $1.38 \pm 0.06$ & $15.16 \pm 0.62$ & $1.53 \pm 0.00$ \\
\hline sPVPAgNPs & $9.19 \pm 1.32$ & $1.17 \pm 0.13$ & $6.40 \pm 0.18$ & $0.94 \pm 0.04$ & $10.66 \pm 0.37$ & $1.43 \pm 0.08$ \\
\hline 1PVPAgNPs & $7.47 \pm 0.37$ & $0.60 \pm 0.20$ & $6.47 \pm 0.15$ & $0.85 \pm 0.71$ & $13.63 \pm 0.35$ & $1.64 \pm 0.04$ \\
\hline CITAgNPs & $11.82 \pm 0.05$ & $1.33 \pm 0.01$ & $7.78 \pm 0.22$ & $0.91 \pm 0.00$ & $10.30 \pm 0.38$ & $1.16 \pm 0.09$ \\
\hline sCITAuNPs & $10.27 \pm 0.35$ & $1.23 \pm 0.02$ & $10.13 \pm 0.37$ & $1.15 \pm 0.03$ & $16.20 \pm 0.74$ & $1.82 \pm 0.04$ \\
\hline mCITtAuNPs & $11.42 \pm 0.23$ & $1.23 \pm 0.10$ & $9.12 \pm 0.50$ & $1.22 \pm 0.15$ & $13.50 \pm 0.24$ & $1.34 \pm 0.03$ \\
\hline 1CITAuNPs & $12.40 \pm 0.46$ & $1.34 \pm 0.03$ & $8.49 \pm 0.18$ & $0.87 \pm 0.08$ & $8.13 \pm 0.42$ & $0.89 \pm 0.06$ \\
\hline PEGAgNPs & $4.75 \pm 0.21$ & $0.54 \pm 0.03$ & $10.48 \pm 0.54$ & $1.22 \pm 0.20$ & $7.24 \pm 0.31$ & $0.87 \pm 0.00$ \\
\hline PEGAuNPs & $9.23 \pm 0.11$ & $1.04 \pm 0.09$ & $11.00 \pm 0.00$ & $1.09 \pm 0.04$ & $9.42 \pm 0.04$ & $0.99 \pm 0.07$ \\
\hline PEGAuNRs & $8.40 \pm 0.50$ & $0.87 \pm 0.04$ & $8.03 \pm 0.41$ & $0.72 \pm 0.05$ & $7.75 \pm 0.98$ & $0.82 \pm 0.06$ \\
\hline sPEGAuNPrs & $9.30 \pm 0.32$ & $0.91 \pm 0.17$ & $9.35 \pm 0.94$ & $0.99 \pm 0.30$ & $10.51 \pm 0.40$ & $1.05 \pm 0.05$ \\
\hline mPEGAuNPrs & $10.61 \pm 0.01$ & $1.02 \pm 0.03$ & $11.25 \pm 0.85$ & $1.42 \pm 0.07$ & $18.32 \pm 1.33$ & $1.82 \pm 0.04$ \\
\hline 1PEGAuNPrs & $12.97 \pm 0.35$ & $1.21 \pm 0.02$ & $11.09 \pm 0.96$ & $1.07 \pm 0.07$ & $11.51 \pm 0.22$ & $1.04 \pm 0.04$
\end{tabular}


Table S3. The Pearson correlation coefficients obtained from the statistical analysis of experimental data. Only correlations of statistical significance are presented.

\begin{tabular}{|c|c|c|c|}
\hline Correlation & All NMs & Spherical NMs & NPrs + NRs \\
\hline Zeta potential vs $\log \mathrm{K}_{\mathrm{b}}(\mathrm{TRF})$ & -0.649925 & -0.682136 & -0.984617 \\
\hline SSA vs $\Delta(\alpha$-helix TRF $)$ & n.s. & n.s. & -0.965475 \\
\hline SSA vs $\Delta(\beta$-sheets TRF $)$ & -0.779494 & -0.808755 & n.s. \\
\hline$\zeta$-potential vs $\log K_{\mathrm{b}}(\mathrm{TRF})$ & -0.649925 & -0.682136 & -0.984617 \\
\hline$\zeta$-potential vs $\Delta(\beta$-sheets TRF $)$ & n.s. & n.s. & -0.961610 \\
\hline$\zeta$-potential vs $\Delta(\beta$-sheets TRF $)$ & n.s. & n.s. & 0.962153 \\
\hline $\log K_{\mathrm{b}}(\mathrm{TRF})$ vs $\Delta(\alpha$-helix TRF $)$ & 0.577909 & n.s. & n.s. \\
\hline$\Delta(\alpha-$ helix BSA $)$ vs $\Delta$ (others BSA $)$ & -0.936138 & -0.937057 & n.s. \\
\hline$\Delta$ ( $\alpha$-helix BSA $)$ vs $\Delta$ ( $\alpha$-helix ngTRF $)$ & 0.838352 & 0.855478 & n.s. \\
\hline$\Delta(\alpha-$ helix BSA $)$ vs $\Delta(\beta$-sheets ngTRF $)$ & n.s. & -0.685899 & n.s. \\
\hline$\Delta$ (others BSA) vs $\Delta$ ( $\alpha$-helix ngTRF $)$ & -0.739938 & -0.764313 & n.s. \\
\hline$\Delta(\alpha-$ helix TRF $)$ vs $\Delta(\beta-$ sheets TRF $)$ & -0.607795 & n.s. & n.s. \\
\hline$\Delta(\beta$-sheets TRF $)$ vs $\Delta$ (others TRF $)$ & -0.560251 & -0.644890 & n.s. \\
\hline$\Delta(\beta$-sheets TRF $)$ vs $\Delta(\alpha-$ helix ngTRF $)$ & n.s. & -0.706703 & n.s. \\
\hline$\Delta$ (others TRF) vs $\Delta$ ( $\beta$-sheets ngTRF) & n.s. & -0.659244 & n.s. \\
\hline$\Delta(\alpha$-helix ngTRF $)$ vs $\Delta(\beta$-sheets ngTRF $)$ & -0.615326 & -0.734578 & -0.960628 \\
\hline
\end{tabular}



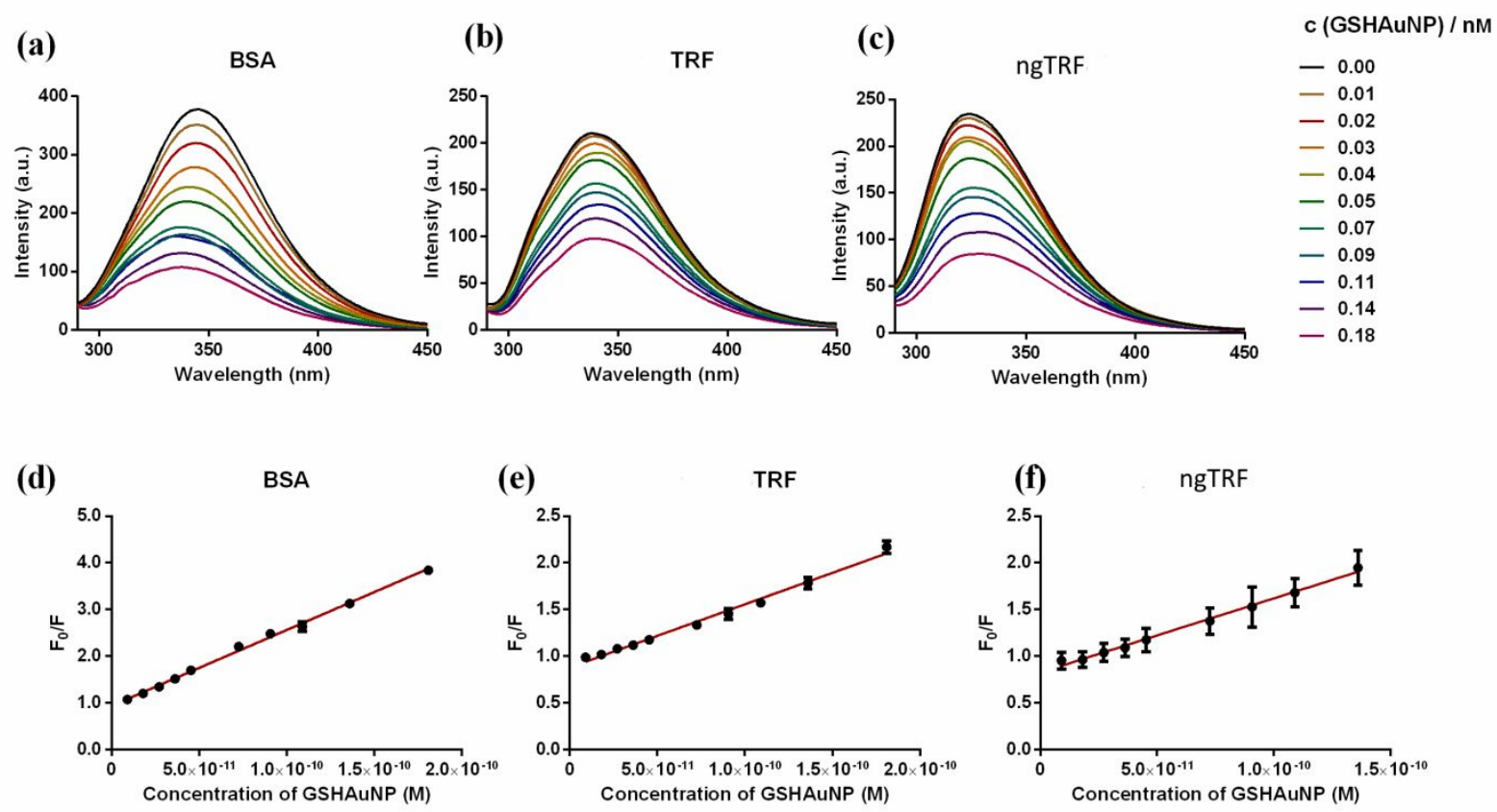

(g)

(h)
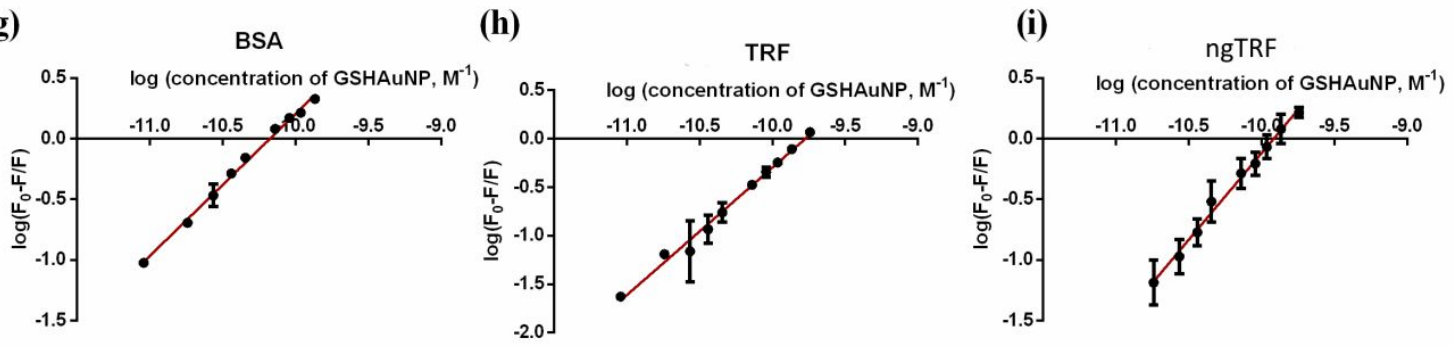

Figure S1. Fluorescence emission spectra of (a) bovine serum albumin (BSA), (b) transferrin (TRF) and (c) non-glycosylated transferrin (ngTRF), obtained at the protein concentration of 0.2 $\mu \mathrm{M}$ in the absence and presence of GSHAuNPs in concentration range of $0.01-0.18 \mathrm{nM}$. SternVolmer plots of fluorescence quenching at $0.2 \mu \mathrm{M}$ (d) BSA (e) TRF and (f) ngTRF by GSHAuNP and corresponding logarithmic plots of the fluorescence quenching of (g) BSA (h) TRF and (i) ngTRF. Error bars represent the standard deviation of the mean of three replicate titrations. 
(a)

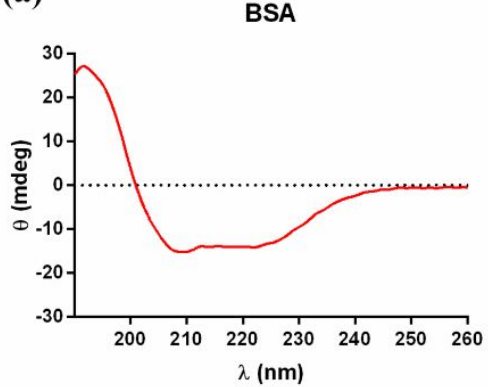

(b)

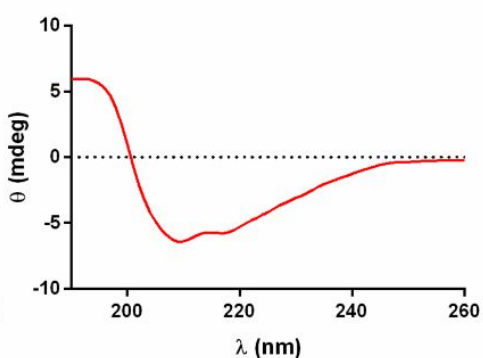

(c)

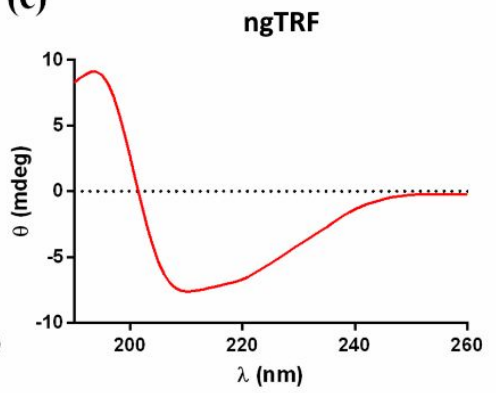

Figure S2. Native conformation of bovine serum albumin (BSA), transferrin (TRF) and nonglycosylated transferrin (ngTRF) recorded using circular dichroism (CD) method.

(a)

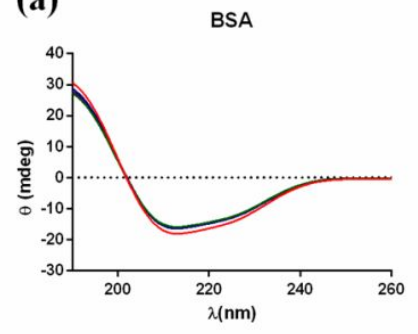

(d)

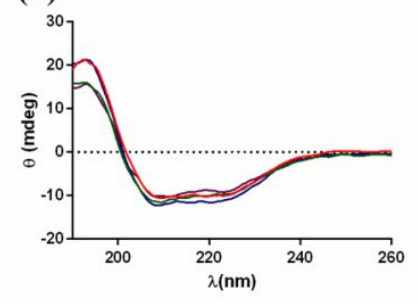

(b)

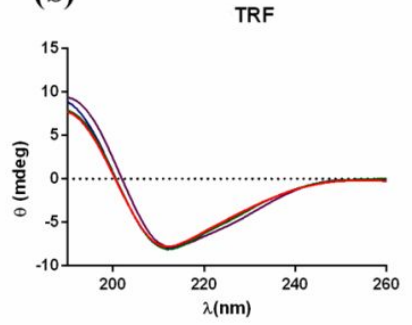

(e)

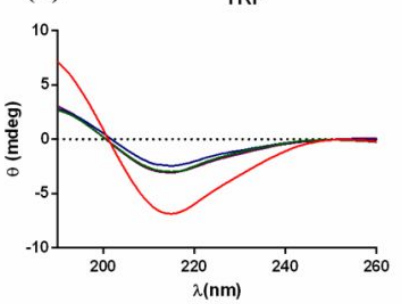

(c)

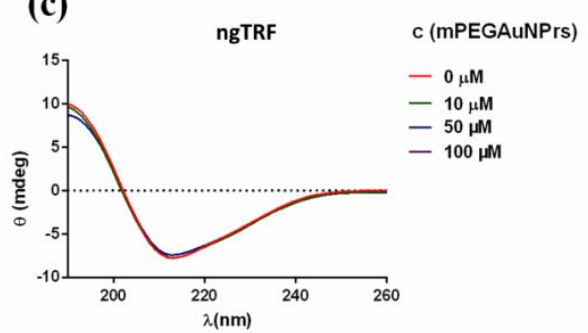

(f)

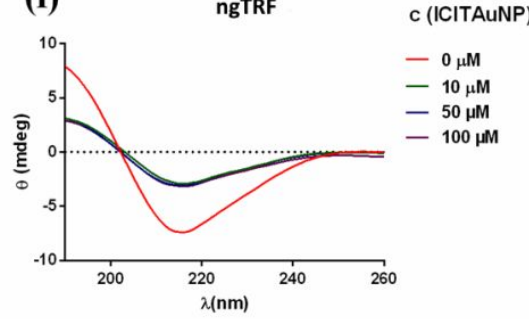

Figure S3. Circular dichroism spectra of bovine serum albumin (BSA), transferrin (TRF) and nonglycosylated transferrin (ngTRF) in the absence and presence of mPEGAuNPrs $(a, b, c)$ and ICITAuNP (d, e, f) at the NPs concentrations of $10 \mu \mathrm{M}, 50 \mu \mathrm{M}$ and $100 \mu \mathrm{M}$. 
(a)

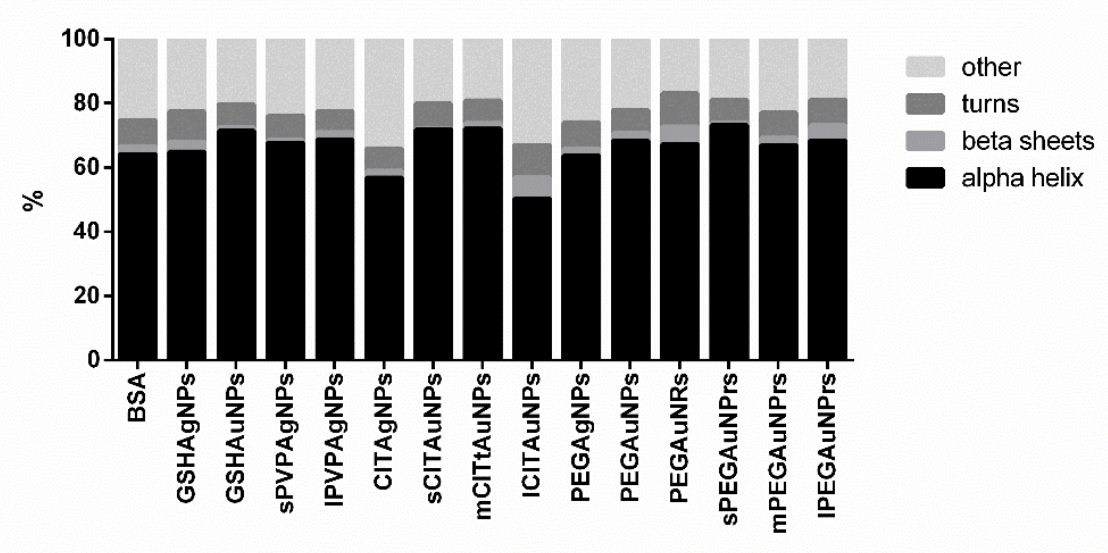

(b)

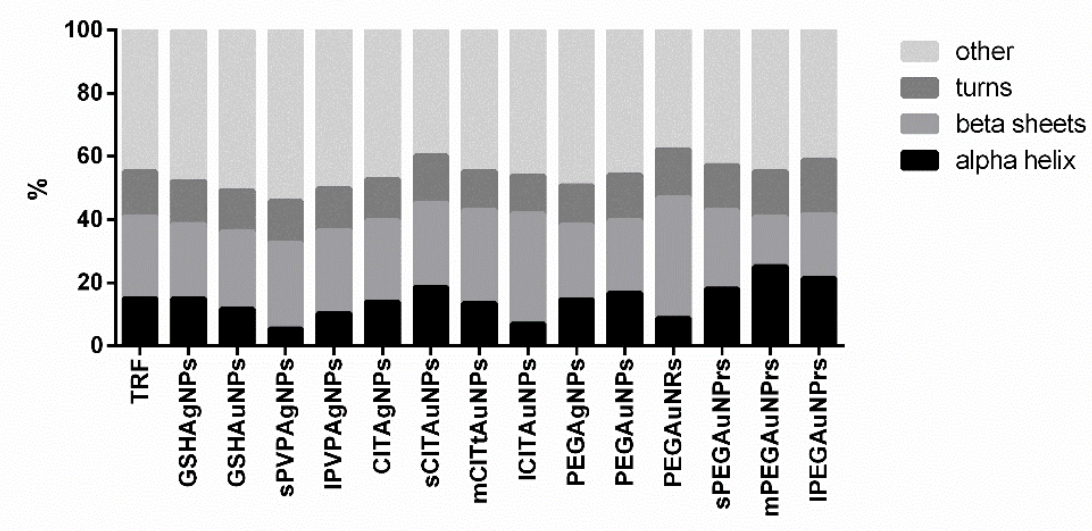

(c)

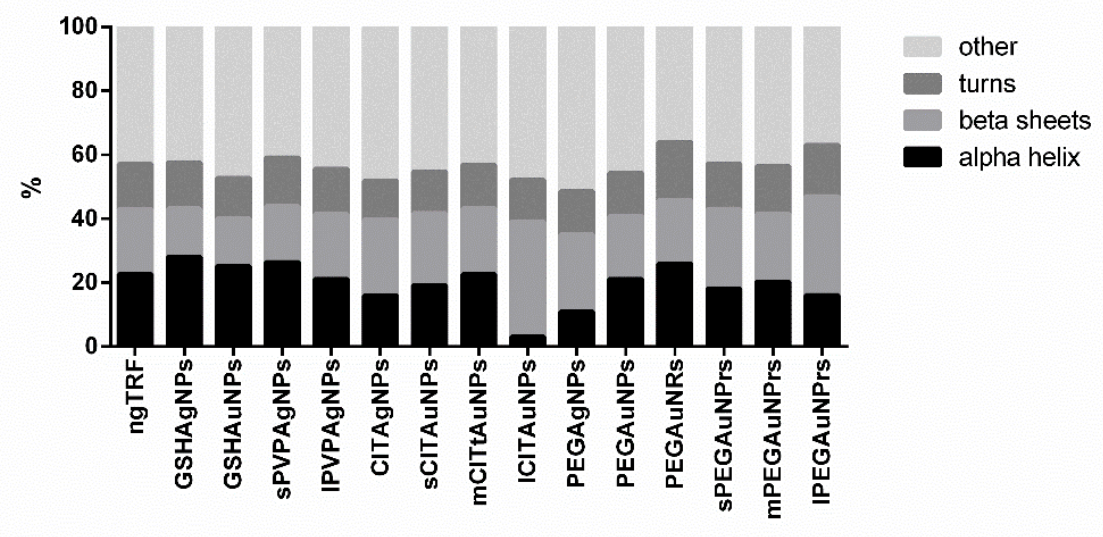

Figure S4. Changes in structural features of (a) bovine serum albumin (BSA), (b) transferrin (TRF) and (c) non-glycosylated transferrin (ngTRF) due to the interaction with different silver and gold nanomaterials at a concentration of $100 \mu \mathrm{M}$ compared to the native protein. 


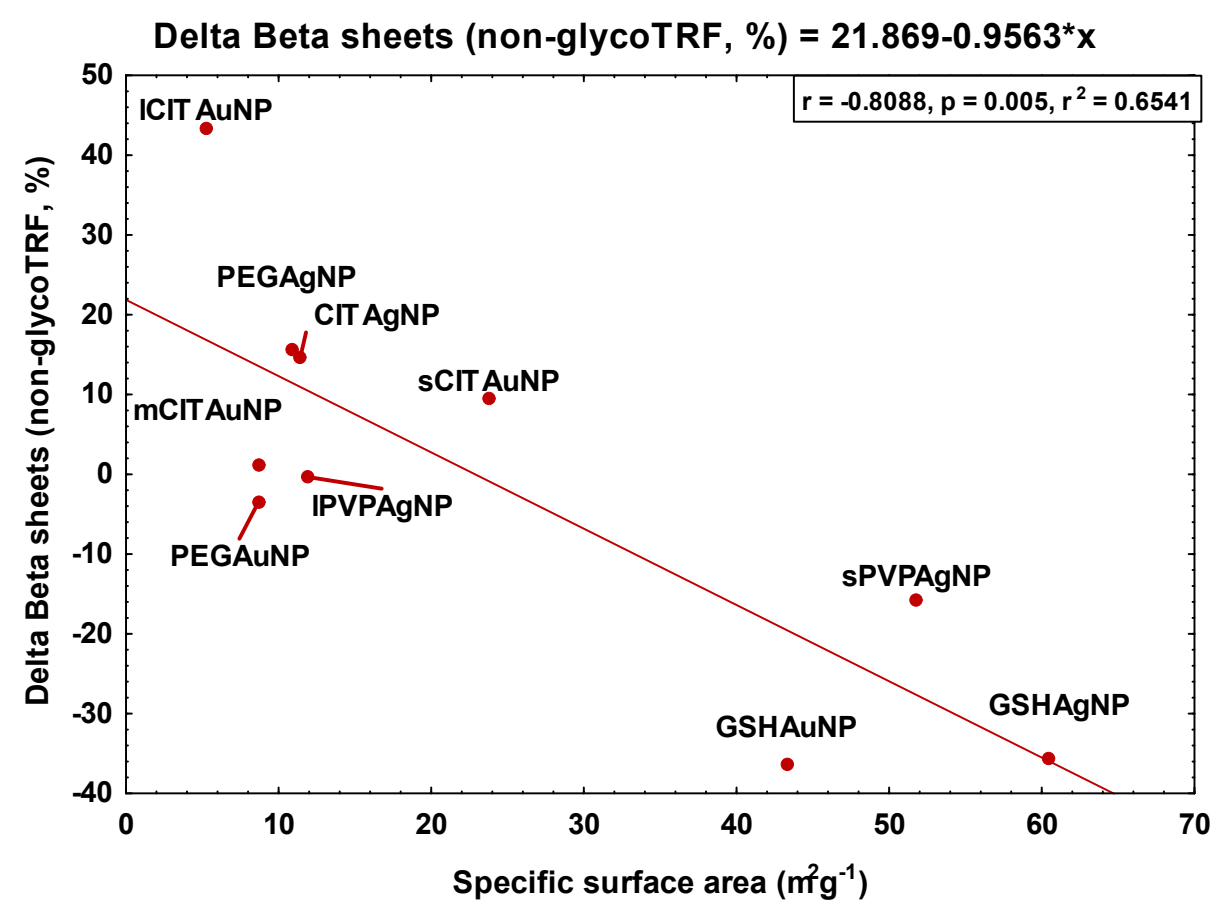

Figure S5. Correlation between delta beta sheets of non-glycoTRF (\%) and specific surface area $\left(\mathrm{m}^{2} \mathrm{~g}^{-1}\right)$ of spherical NPs. 